\title{
Michelson wide-field stellar interferometry: principles and experimental verification
}

\author{
I. Montilla, S. F. Pereira, and J. J. M. Braat
}

\begin{abstract}
A new interferometric technique for Michelson wide-field interferometry is presented that consists of a Michelson pupil-plane combination scheme in which a wide field of view can be achieved in one shot. This technique uses a stair-shaped mirror in the intermediate image plane of each telescope in the array, allowing for simultaneous correction of the differential delay for the on-axis and off-axis image positions. Experimental results in a laboratory setup show that it is possible to recover the fringes of on-axis and off-axis stars with an angular separation of 1 arc min simultaneously and with a similar contrast. This new technique represents a considerable extension of the field of view of an interferometer without the need for extra observation time. (C) 2005 Optical Society of America

OCIS codes: $\quad 120.3180,350.1260,350.1270,120.0120,260.3160$.
\end{abstract}

\section{Introduction}

Optical stellar interferometry ${ }^{1}$ consists of using two or more telescopes that collect light from a distant object to produce information with high angular resolution. When two apertures are separated by a distance $B$, called the baseline, the flat wave front that originates from the distant source at a certain angle $\theta$ from the baseline does not reach the apertures simultaneously (see Fig. 1). This delay will introduce an optical path-length difference (OPD) between the two arms of the interferometer. If the OPD is larger than the coherence length, the light from the two apertures will not interfere. ${ }^{2}$ To ensure that interference will occur, an extra path in one of the arms of the interferometer should be introduced. When the interferometer is not of the Fizeau type, ${ }^{3}$ an increase in resolution implies a decrease in the field of view. The reason is that the OPD is corrected for the pointing direction of the telescope.

A wide field of view is important in applications such as observation of extended or multiple objects or fringe acquisition and tracking on a nearby unresolved object ${ }^{4}$ and also for reducing the observation

The authors are with the Imaging Science and Technology Department, Optics Research Group, Delft University of Technology, P.O. Box 5046, NL-2600 GA Delft, The Netherlands. I. Montilla's e-mail address is montilla@optica.tn.tudelft.nl.

Received 27 April 2004; revised manuscript received 7 October 2004; accepted 7 October 2004.

0003-6935/05/030328-09\$15.00/0

(C) 2005 Optical Society of America time. In homothetic mapping the synthetic aperture of the telescope array is exactly reproduced in a downscaled version at the recombination optics; this recombination scheme has intrinsic path-length compensation and a correspondingly wide field. Therefore this scheme is the standard wide-field method, but when the aperture is highly diluted its applicability is limited by the signal-to-noise ratio. Fizeau-type interferometers present the problems that the complexity of the system increases with the field-to-resolution ratio, which is the number of resolved elements in the desired field, ${ }^{5}$ and that the signal-to-noise ratio decreases with the number of telescopes, limiting the number of baselines that can be used in a system. ${ }^{6}$ When the aperture is highly diluted, the central interference peak contains only a small fraction of the photons ${ }^{7}$; the rest of the energy is dispersed in the diffractive halo.

To increase the signal-to-noise ratio, Labeyrie proposed densification of the exit pupil. 8 This consists of increasing the exit apertures' diameter by a factor called the pupil concentration coefficient while maintaining their centers' position. Experimental results obtained by Pedretti et al. ${ }^{9}$ showed an intensity gain of $24 \pm 3 \times$ when a densified array was compared with an equivalent Fizeau-type interferometer.

Coaxial combination is an extreme case of densification. Pupil-plane interferometers or densified arrays have the disadvantage that the field of view consists only of a few resolution elements. Of course, direct images cannot be obtained when the pupils are overlapped, but the gain in intensity is the highest possible. To use this property we thought of a new 


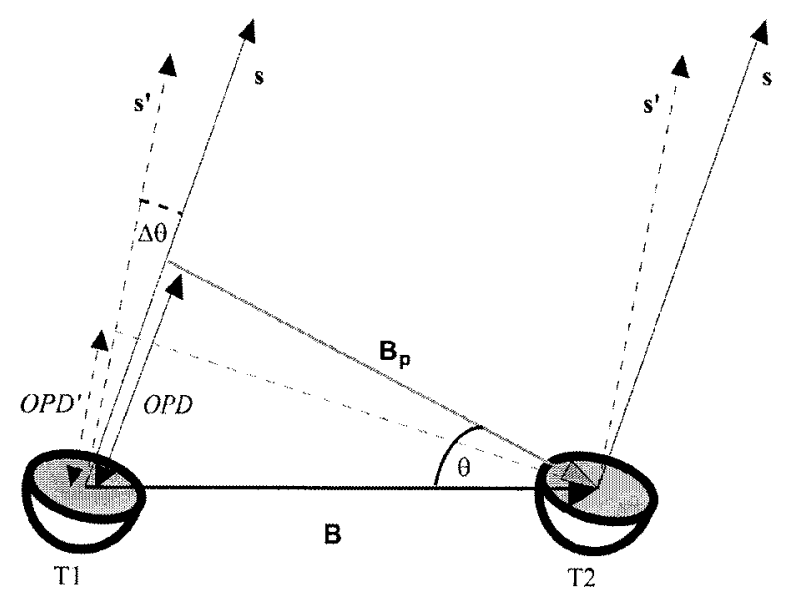

Fig. 1. OPD as a function of field angle. The differential OPD depends not only on baseline $\mathbf{B}$ and angle $\Delta \theta$ between the pointing direction $\mathbf{s}$ and another direction in the sky $\mathbf{s}^{\prime}$ but also on angle $\theta$ between $\mathbf{s}$ and $\mathbf{B}$.

approach to the problem, i.e., a system that could use a Michelson pupil-plane combination scheme to acquire a wide field of view in one shot, thus also saving observation time. Based on these ideas, we introduce a new technique, called a staircase mirror approach, which consists of positioning a stair-shaped mirror into an intermediate image plane for each telescope in the array. In this way, the light from different stars is imaged on different steps on the mirror, permitting simultaneous correction of the OPD. The shape of the mirror depends on the baseline and on the pointing direction, and it must be determined during the observation, as the entrance pupil varies with hour angle. ${ }^{10}$ This paper is organized as follows: in Section 2 we derive the equation for the OPD as a function of the position in the field. We have adopted here the celestial coordinates that are commonly used in astronomical observations. The reader who is interested primarily in the way in which our method works can skip the details of the coordinate transformation in this section. In Section 3 we calculate the shape of the mirror required for correction of the differential OPD and explain how it must be actuated to permit correction during the observation, and in Section 4 we show its application to a particular case, that of the Very Large Telescope Interferometer (VLTI). ${ }^{11}$ The experimental setup is described in Section 5, and measurements that demonstrate the feasibility of the concept are addressed in Section 6 .

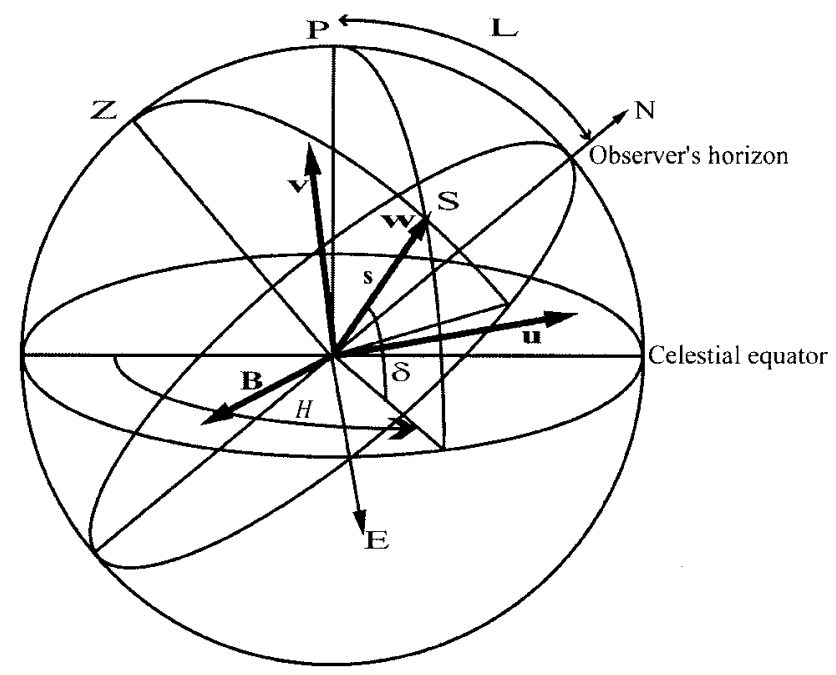

Fig. 2. Local horizon coordinate system $\mathbf{X Y Z}$, where baseline $\mathbf{B}$ is defined, and coordinate system $\mathbf{u v w}$, where $\mathbf{s}$ and $\mathbf{s}^{\prime}$ are defined. The $\mathbf{X Y}$ plane is the observer's horizon, north is the $\mathbf{X}$ direction, east is the $\mathbf{Y}$ direction, and $\mathbf{Z}$ is the zenith. The latitude of the observing site is $L$. In the $\mathbf{u v w}$ system $\mathbf{w}$ is parallel to $\mathbf{s}, \mathbf{u}$ is perpendicular to the plane defined by $\mathbf{w}$ and pole $P$, and $\mathbf{v}$ is perpendicular to the plane defined by $\mathbf{u}$ and $\mathbf{w}$.

\section{Optical Path Difference Dependence on Field}

The extra external OPD for any off-axis direction has been calculated as a function of the pointing direction and the baseline vector as shown in Fig. 2. We consider an object $\mathrm{S}$, in a position given by $\mathbf{S}$ with celestial coordinates in the equatorial system $^{12} \delta$ (declination) and $H$ (hour angle). Two telescopes, T1 and $\mathrm{T} 2$, separated by a distance $B$, are positioned at the origin and at the end, respectively, of baseline vector $\mathbf{B}$. The declination of a vector $\mathbf{s}^{\prime}$, which is slightly different from the pointing direction, can be represented as $\delta+\Delta \delta$ with hour angle $H+\Delta \mathrm{H}$. The coordinates of baseline vector $\mathbf{B}$ are defined in the local horizon Cartesian system: the $\mathbf{X Y}$ plane is the observer's horizon, and north is the $\mathbf{X}$ direction. The $\mathbf{Z}$ direction points to the zenith. The scalar product $(\mathbf{B} \cdot \mathbf{s})$ is the OPD. To calculate this scalar product we express $\mathbf{B}$ and $\mathbf{s}$ in the same coordinate system. We define a new Cartesian system $\mathbf{u v w}$, where $\mathbf{w}$ is parallel to $\mathbf{s}, \mathbf{u}$ is perpendicular to the plane defined by $\mathbf{w}$ and the pole $(\mathrm{P})$ and points toward the positive hour angle direction, and $\mathbf{v}$ is perpendicular to the plane defined by $\mathbf{u}$ and $\mathbf{w}$. The following rotation matrix gives the change from $\mathbf{X Y Z}$ to $\mathbf{u v w}$ coordinates:

$$
R=\left[\begin{array}{ccc}
\cos H & \sin H \sin L & -\sin H \cos L \\
-\sin \delta \sin H & \sin \delta \cos H \sin L+\cos \delta \cos L & -\sin \delta \cos H \cos L+\cos \delta \sin L \\
\cos \delta \sin H & -\cos \delta \cos H \sin L+\sin \delta \cos L & \cos \delta \cos H \cos L+\sin \delta \sin L
\end{array}\right]
$$


where $L$ is the latitude of the site from which we are observing. The coordinates of $\mathbf{B}$ in the uvw coordinate system are

$$
\left[\begin{array}{l}
B_{u} \\
B_{v} \\
B_{w}
\end{array}\right]=\mathbf{R}\left[\begin{array}{l}
B_{x} \\
B_{y} \\
B_{z}
\end{array}\right],
$$

and the coordinates of $\mathbf{s}$ are

$$
\left[\begin{array}{l}
s_{u} \\
s_{v} \\
s_{w}
\end{array}\right]=\left[\begin{array}{l}
0 \\
0 \\
1
\end{array}\right] .
$$

As can be seen from Fig. 1, the OPD and $\mathrm{OPD}^{\prime}$ at T1 with respect to $\mathrm{T} 2$ for the directions given by $\mathbf{s}$ and $\mathbf{s}^{\prime}$ are, respectively:

$$
\begin{aligned}
\mathrm{OPD}= & B_{w}=B_{x} \cos \delta \sin H+B_{y}(\cos L \sin \delta \\
& -\sin L \cos \delta \cos H)+B_{z}(\cos \delta \cos H \cos L \\
& +\sin \delta \sin L),
\end{aligned}
$$

$$
\begin{aligned}
\mathrm{OPD}^{\prime}= & B_{w}{ }^{\prime}=B_{x} \cos (\delta+\Delta \delta) \sin (H+\Delta \mathrm{H}) \\
& +B_{y}[\cos L \sin (\delta+\Delta \delta)-\sin L \cos (\delta \\
& +\Delta \delta) \cos (H+\Delta \mathrm{H})]+B_{z}[\cos (\delta+\Delta \delta) \\
& \times \cos (H+\Delta H) \cos L+\sin (\delta+\Delta \delta) \sin L) .
\end{aligned}
$$

The projection of $\mathbf{B}$ onto the entrance pupil plane of $\mathrm{T} 1$ is

$$
\mathbf{B}_{p}=\left[\begin{array}{c}
B_{u} \\
B_{v} \\
0
\end{array}\right] .
$$

The difference between OPD ${ }^{\prime}$ and OPD is the differential OPD, or differential delay, $\Delta \mathrm{OPD}_{\mathrm{ext}}=B_{w}{ }^{\prime}$ $-B_{w}$. Expanding the sines and cosines in Eq. (5) and considering only the first-order terms of $\Delta \mathrm{OPD}_{\text {ext }}$, we obtain the following expression:

$$
\begin{aligned}
\Delta \mathrm{OPD}_{\mathrm{ext}}= & \Delta \delta\left[-B_{x} \sin \delta \sin H+B_{y}(\cos L \cos \delta\right. \\
& +\sin L \sin \delta \cos H) \\
& \left.-B_{z}(\sin \delta \cos H \cos L-\cos \delta \sin L)\right] \\
& +\Delta \mathrm{H}\left(B_{x} \cos \delta \cos H\right. \\
& +B_{y} \sin L \cos \delta \sin H \\
& \left.-B_{z} \cos \delta \sin H \cos L\right) .
\end{aligned}
$$

In practice, a linear approximation of $\triangle \mathrm{OPD}_{\text {ext }}$ is valid because, in general, for a field of view of several arc minutes the second-order terms of the expression are several orders of magnitude smaller than the firstorder terms. For example, for the typical parameters used by some of the VLTI instruments (baseline,

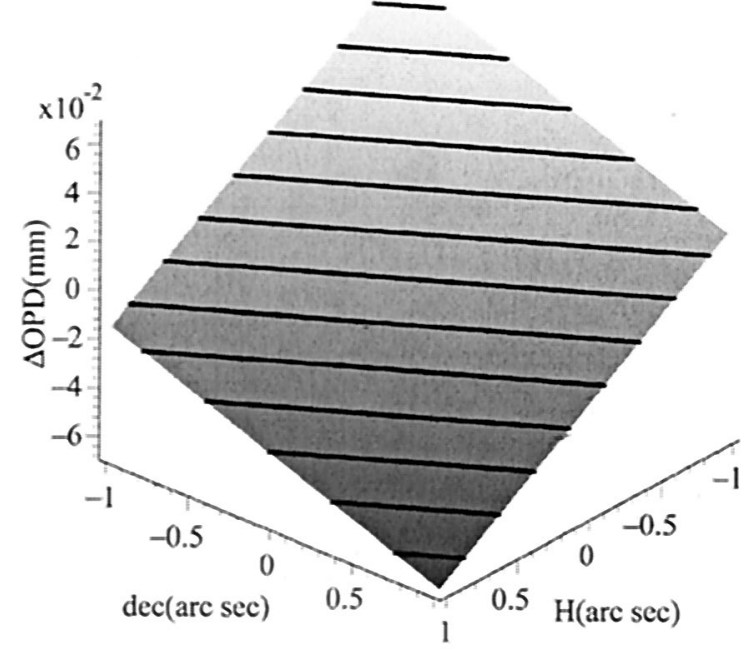

Fig. 3. Differential OPD shape; the contour lines are lines of constant OPD.

$200 \mathrm{~m}$; wavelength, $2.2 \mu \mathrm{m}$; bandwidth, $0.22 \mu \mathrm{m}$ ) correcting only the first-order terms yields a cophased field of view (where the OPD errors are smaller than the wavelength) of $1 \mathrm{arc}$ min and a coherenced field of view (where the OPD errors are smaller than the coherence length) of 2 arc min.

We can see that the function that represents $\Delta \mathrm{OPD}_{\text {ext }}$ depends not only on the baseline vector but also on the declination and the hour angle of the pointing vector. The shape of the linearized $\Delta O P D_{\text {ext }}$ function is a tilted plane where the constant OPD zones, shown in Fig. 3, are perpendicular to $\mathbf{B}_{p}$. If $\mathbf{B}$ points from $\mathrm{T} 1$ to $\mathrm{T} 2$, the projection of the baseline in the entrance pupil is parallel to the gradient of $\Delta \mathrm{OPD}_{\text {ext }}$ at $\mathrm{T} 1$.

\section{Optical Path Difference Correction: Staircase Mirror}

The first-order corrections for the OPD can be introduced by means of a staircase mirror situated in the focal plane of the optical subsystems before the beam combination. The focal plane has the characteristic that the light from different parts of the sky is focused separately (Fig. 4); that is why we intend to use it to introduce the equalization of the OPD. The correction introduced by one step is

$$
\Delta \mathrm{OPD}=2 d \cos \alpha,
$$

where $\alpha$ is the angle between the normal to the mirror surface and the optical axis of the system and $d$ is the depth of the step. In an intermediate focal plane, with focal length $F$, the angular coordinates $\Delta H$ and $\Delta \delta$, expressed in radians, correspond to focal-plane coordinates $u=t \Delta H$ and $v=t \Delta \delta$, where $t$ is the scale in the focal plane, in meters per radian. Using this conversion and Eqs. (7) and (8), we can derive the equation of the planar surface $\mathrm{OPD}_{\text {int }}$, to which the 
shape of the mirror has to be adjusted, to correct $\Delta \mathrm{OPD}_{\text {ext }}$ in the focal plane:

$$
\begin{aligned}
\mathrm{OPD}_{\text {int }}= & \frac{1}{2 t \cos \alpha}\left\{u \left[-B_{x} \sin \delta \sin H\right.\right. \\
& +B_{y}(\cos L \cos \delta+\sin L \sin \delta \cos H) \\
& \left.-B_{z}(\sin \delta \cos H \cos L-\cos \delta \sin L)\right] \\
& +v\left(B_{x} \cos H+B_{y} \sin L \sin H\right. \\
& \left.\left.-B_{z} \sin H \cos L\right)\right\}
\end{aligned}
$$

where $B_{x}$ and $B_{y}$ are the coordinates of the telescope in the local horizon system, in meters, from an external reference point.

The mirror consists of steps of fixed width and adaptable depth. The depth of the steps must vary, as it has to be adapted to the changes in $B_{w}$. The mirror has to be rotated to follow the rotation of $\mathbf{B}_{p}$ to have the steps perpendicular to it. As we explained, $\mathbf{B}_{p}$ is parallel to the gradient of the $\mathrm{OPD}_{\text {int }}$ function. This property will determine the relation between the width of the steps, $w$, and the depth, $d$. The gradient of the $\mathrm{OPD}_{\text {int }}$ function is given by the vector $\nabla O P D_{\text {int }}=\left(\partial \mathrm{OPD}\right.$ int $\left./ \partial u, \partial \mathrm{OPD}_{\text {int }} / \partial v\right)$. The width of the steps is set equal to the modulus of a vector $p$ $=a \nabla \mathrm{OPD}_{\text {int }}$, where $a$ is a distance in meters and is given by

$$
w=a\left[\left(\frac{\partial \mathrm{OPD}_{\mathrm{int}}}{\partial u}\right)^{2}+\left(\frac{\partial \mathrm{OPD}_{\mathrm{int}}}{\partial v}\right)\right]^{1 / 2} .
$$

The depth of the steps is equal to the value of the function $\mathrm{OPD}_{\text {int }}$ at the point determined by the coordinates of $\mathbf{p}$ :

$$
d=\nabla \mathrm{OPD}_{\text {int }} \cdot \mathbf{p}=a\left[\left(\frac{\partial \mathrm{OPD}_{\text {int }}}{\partial u}\right)^{2}+\left(\frac{\partial \mathrm{OPD}_{\text {int }}}{\partial v}\right)^{2}\right]^{1 / 2} .
$$

Therefore the width and the depth of the mirror are related by the following equation:

$$
\frac{w}{d}=\left[\left(\frac{\partial \mathrm{OPD}_{\text {int }}}{\partial u}\right)^{2}+\left(\frac{\partial \mathrm{OPD}_{\text {int }}}{\partial v}\right)^{2}\right]^{-1 / 2} .
$$

As the width of the steps is fixed, it has to fulfill two conditions: It has to be bigger than one Airy disk such that the standard interferometric field of view is not cut and it has to be valid for correcting the OPD at any pointing direction. We calculate $w$ as follows: The maximum depth, which is a function of coherence length $L_{c}$ and angle $\alpha$, depends on the maximum optical path that we want to scan. If the maximum path that we want to scan is 1.5 times the coherence length, then the maximum depth is given by $d_{\max }$ $=3 L_{c} /(2 \cos \alpha)$. Then, given the values of the maximum depth and the baseline, we calculate the minimum width for any pointing direction, $w_{\min }$, and check whether it is effectively bigger than one Airy

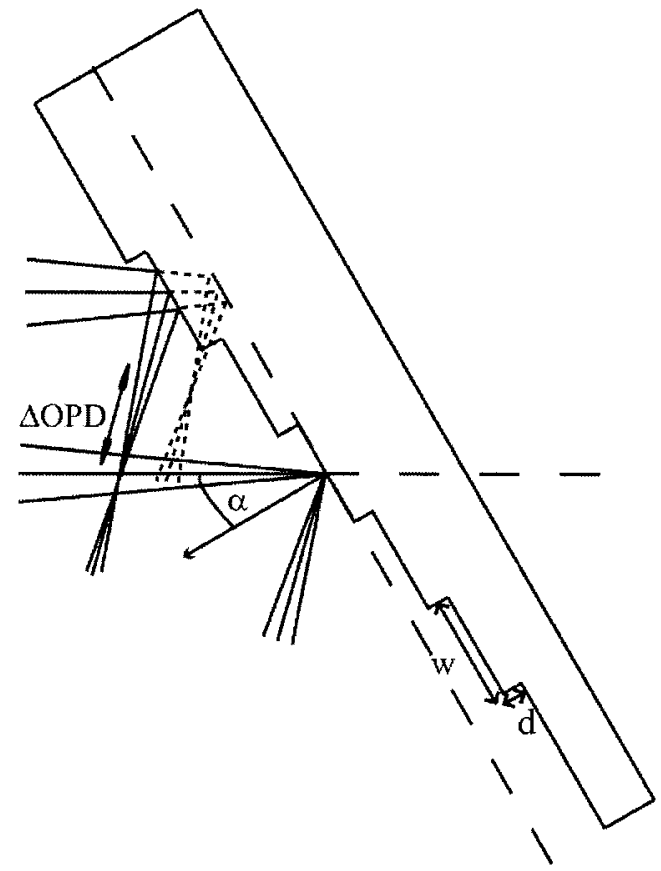

Fig. 4. OPD equalization; the light from different directions in the field is focused on different steps, and this effect introduces an extra OPD that depends on the field angle.

disk. If it is not, we choose a larger $d_{\max }$. The position of the minimum is given by $\delta_{\min }$ and $H_{\min }$, which are solutions of the following system:

$$
\begin{gathered}
\frac{\partial w}{\partial \delta}\left(\delta_{\min }, H_{\min }\right)=0, \\
\frac{\partial w}{\partial H}\left(\delta_{\min }, H_{\min }\right)=0, \\
\frac{\partial^{2} w}{\partial \delta \partial H}\left(\delta_{\min }, H_{\min }\right)>0 .
\end{gathered}
$$

With width $w_{\min }=w\left(\delta_{\min }, H_{\min }\right)$, the expression for $B_{p}$, and the equation for $\mathrm{OPD}_{\text {int }}$, we can write the function $M(u, v)$ that rules the shape of a mirror, with $(2 N+1)$ steps, as follows:

$$
M(u, v)=\sum_{n=-N}^{N} n d \operatorname{rect}\left(\frac{B_{u} u+B_{v} v}{w_{\min } B_{p}}-n\right),
$$

with the function $\operatorname{rect}(x)$ given by $\operatorname{rect}(x)=1$ when $|x| \leq 1 / 2$ and zero elsewhere and

$$
d=w_{\min }\left[\left(\frac{\partial \mathrm{OPD}_{\mathrm{int}}}{\partial u}\right)^{2}+\left(\frac{\partial \mathrm{OPD}_{\mathrm{int}}}{\partial v}\right)^{2}\right]^{1 / 2}
$$

In a system consisting of more than two telescopes it is necessary to have a step mirror at the focal plane of each telescope. We need a common reference point for the different baselines, and the line parallel to the edge of the steps of each mirror should be perpendic- 


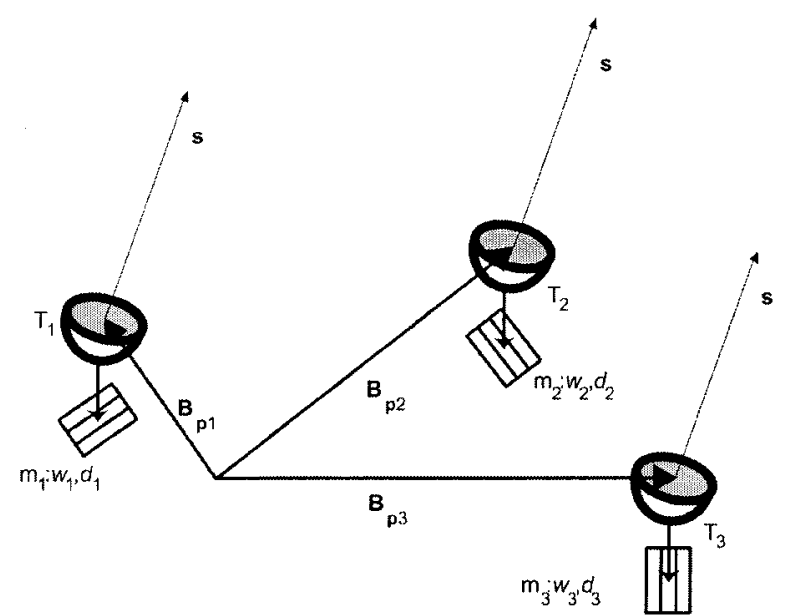

Fig. 5. Mirror position for a telescope system. Telescopes $\mathrm{T}_{1}, \mathrm{~T}_{2}$, and $\mathrm{T}_{3}$ have mirrors $m_{1}, m_{2}$, and $m_{3}$ with widths $w_{1}, w_{2}$, and $w_{3}$ and depths $d_{1}, d_{2}$, and $d_{3}$, respectively. The dividing lines of each staircase mirror should be perpendicular to the projection of each baseline on the entrance pupil of each telescope, $\mathbf{B}_{\mathrm{p} 1}, \mathbf{B}_{\mathrm{p} 2}$, and $\mathbf{B}_{\mathrm{p} 3}$, defined by pointing vector $\mathbf{s}$.

ular to the projection of the baseline on the entrance pupil of the particular telescope, as shown in Fig. 5.

\section{Application To a Real Case: the Very Large Telescope Interferometer}

We have calculated the shape of the mirror that would be required for correction of $\triangle \mathrm{OPD}_{\text {ext }}$ in the Coudé focus of unit telescope 2 (UT2) in the VLTI. In the VLTI system the coordinates of the telescopes are given with respect to a common reference point. From this reference point the reference baseline of UT2 is $24 \times 2^{1 / 2} \mathrm{~m}$. For a wavelength of $2.2 \mu \mathrm{m}$ and a bandwidth of $0.22 \mu \mathrm{m}$, the coherence length of the light is $22 \mu \mathrm{m}$. In this case we consider the telescopes to be coplanar, and we therefore dismiss the term given by $B_{z} \cdot{ }^{13}$ If the normal of the mirror makes an angle of $60^{\circ}$ with the optical axis, the maximum depth of the steps is $66 \mu \mathrm{m}$. With this depth, we plot the width as it depends on the declination and hour angle, as shown

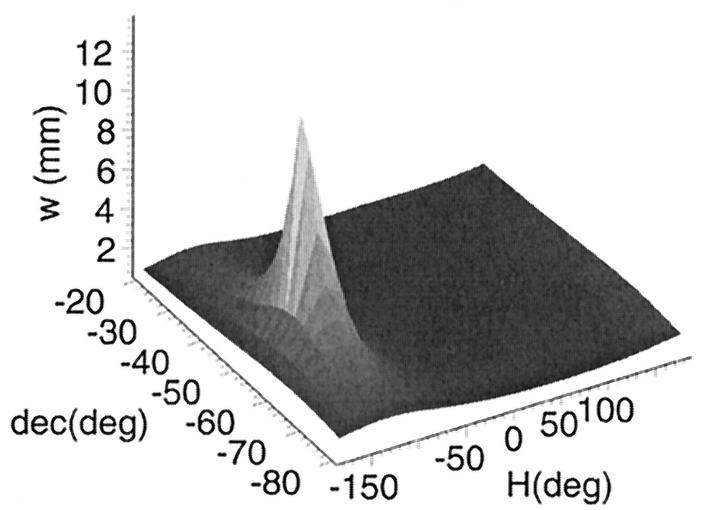

Fig. 6. Mirror width as a function of declination and hour angle for a given baseline and a maximum depth. The areas with low $w$ values correspond to areas of low gradient of the OPD function.

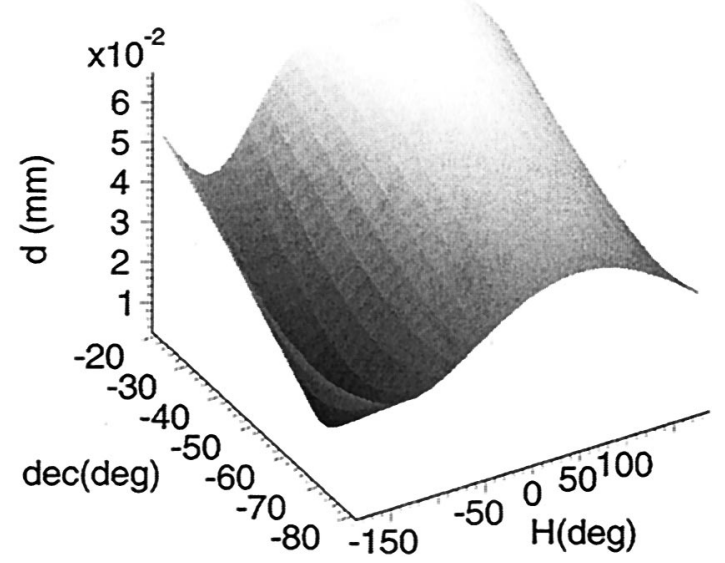

Fig. 7. Mirror depth as a function of declination and hour angle for a given baseline and a minimum width.

in Fig. 6. We take the minimum of this function as the width of the steps. For UT2 the minimum width is $802 \mu \mathrm{m}$. With this width, we plot the depth of the steps as a function of declination and hour angle (see Fig. 7). Note that this function never takes a value larger than $66 \mu \mathrm{m}$ for any direction in the sky.

We now point UT2 to an object situated at declination $\delta=50^{\circ} 47^{\prime} 1^{\prime \prime}$ and right ascension. Celestial objects are at constant RA but change their hour angles $H$ as time proceeds; the relation between them is $H$ $=\mathrm{LST}-\mathrm{RA}$, where LST is the local sidereal time. ${ }^{12}$ Using a mirror with $w=802 \mu \mathrm{m}$, we find a variation of the depth as a function of LST and the rotation of $\mathbf{B}_{p}$ as in Figs. 8(a) and 8(b), respectively. The shape of the mirror is shown in Fig. 9 at three moments during the observation, with a difference of $3 \mathrm{~h}$ between them.

\section{Description of the Experimental Setup}

We have designed and implemented a tabletop setup consisting of a two-telescope Michelson-type interferometer. In the focal plane of one of the arms of the interferometer a staircase mirror was placed. A schematic of the setup is plotted in Fig. 10; the setup consists of three main blocks: a star simulator, an interferometer, and a beam combiner.

The star simulator consists of a xenon-arc lamp with a set of filters that reduces its spectrum to a bandwidth of $150 \mathrm{~nm}(500-650 \mathrm{~nm})$, resulting in a coherence length of $2.2 \mu \mathrm{m}$. The light is imaged onto the star mask, which consists of a set of $5-\mu \mathrm{m}$ pinholes, where each pinhole defines one star. In the experiment described here the illuminated mask has four pinholes, distributed in a square pattern with a separation of $150 \mu \mathrm{m}$. The size of the holes ensures full spatial coherence for the beams over the entire bandwidth. The light from two pinholes is not coherent, ensuring that there are two independent sources, as is the case with real stars. The light from the star mask is collimated in such a way that in the entrance pupil of the telescopes we have four beams, simulat- 


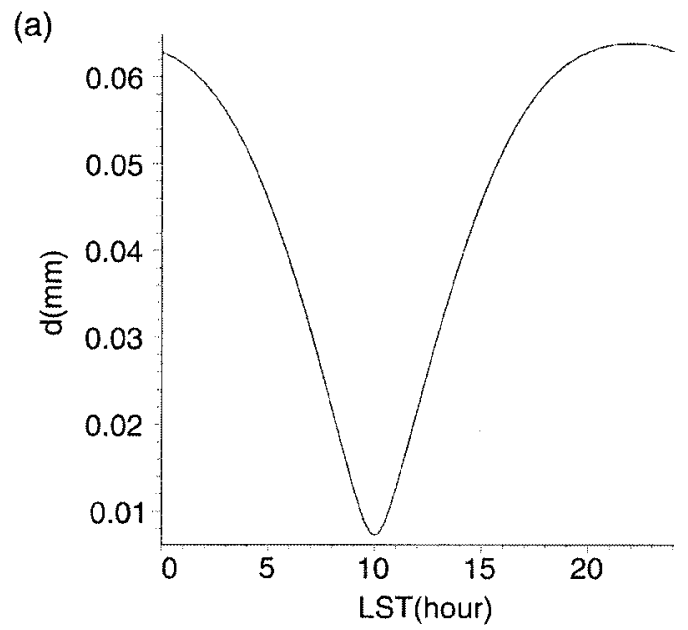

(b)

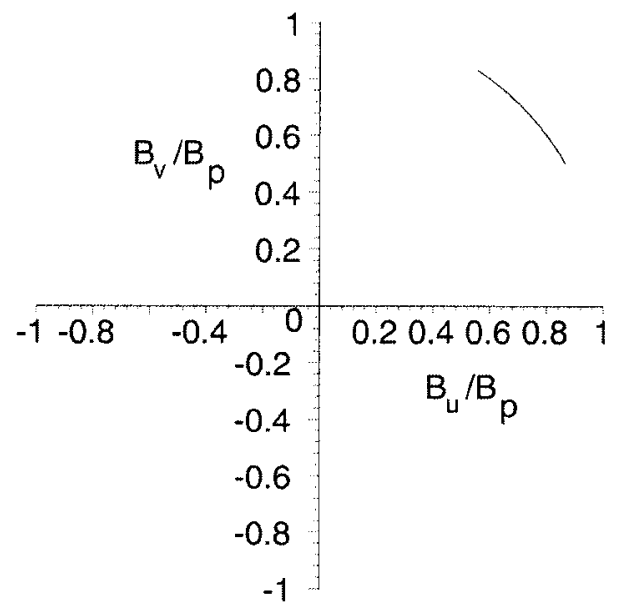

Fig. 8. (a) Mirror depth plotted as a function of the LST for a given width, declination, and baseline. (b) Projection of the baseline vector on the entrance pupil. Because of the rotation of the Earth, the telescope has to change its pointing direction to follow an object in the sky. The result is the rotation of $\mathbf{B}_{\mathrm{p}}$ during the observation.

ing four distant objects in the sky. Two of these objects have external OPDs equal to zero; therefore they are considered on-axis objects. The other two have external OPDs different from zero; hence for the interferometer they are off-axis objects, with an off-axis angle of 1.03 arc min.

The two interferometer beams are obtained by wave-front division by means of two apertures of 20-mm diameter, and the separation between the apertures (i.e., the baseline) is $30 \mathrm{~mm}$. After each aperture a telescope objective is placed, and at the focal plane of one of the interferometer arms a staircase mirror is set, such as drawn in Fig. 4. The staircase mirror introduces a temporal delay to the imaged spots that depends on the incidence angle of the incoming beam at the entrance pupil of the telescope. The introduced delay depends on the tilt angle of the mirror. We chose a tilt of $45^{\circ}$ such that the symmetry between the two arms is easily maintained. The width of the step depends on the effective focal length, $F$, of the telescope objective. It was chosen (a)

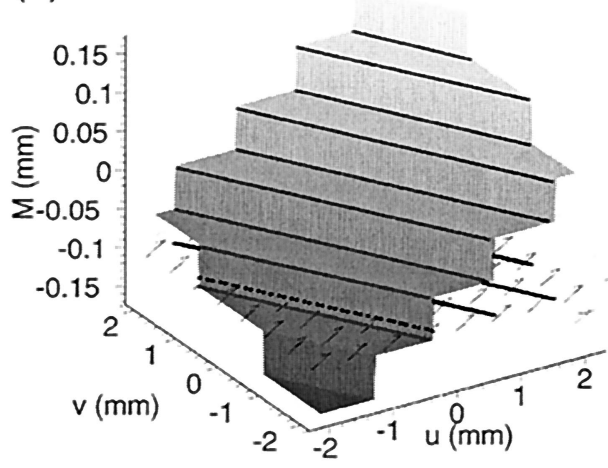

(b)

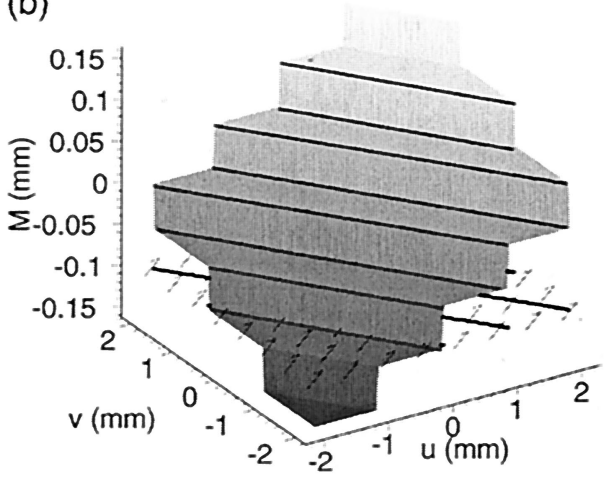

(c)

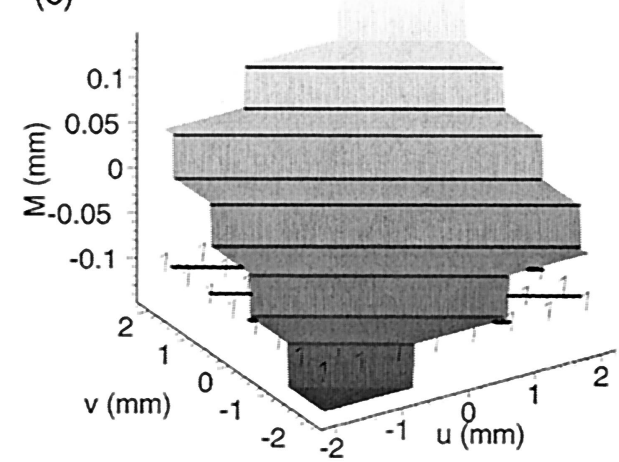

Fig. 9. Simulation of a staircase mirror placed on the Coudé focus of UT2. The shape of the mirror is shown for three different values of LST, (a), (b), and (c), at intervals of $3 \mathrm{~h}$. The uv plane is the focal plane. The lines are the projections of the mirror steps on the uv plane. The faint arrows are gradients of the OPD and are perpendicular to the steps. The baseline projected in the entrance pupil is parallel to the gradient and points in the same direction. During observation, as the projection of the baseline on the entrance pupil rotates, the mirror follows this movement.

such that the point-spread function of the focused spot is several times smaller than the width of the step. In the actual configuration the effective focal length is $6.563 \mathrm{~m}$, and the system is free of lateral and axial chromatic aberration. The depth of the step, $d$, is related to the width, $w$, and the tilt angle, $\alpha$, by the equation

$$
\frac{d}{w}=\frac{B}{2 F} \tan \alpha
$$




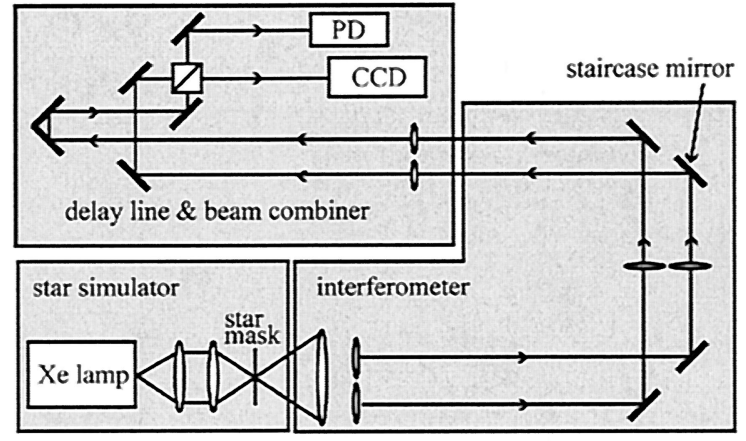

Fig. 10. Schematic of the experimental setup consisting of three blocks: a star simulator with a white-light source and a star mask; an interferometer with the staircase mirror in one of the arms; and a beam combiner with a delay line. A detector was placed in one of the outputs of the beam splitter, and a CCD was placed at the other output to control pupil position. The delay line was connected to a piezo driver (PD) and to a signal generator to modulate the path, producing temporal fringes.

where $B$ is the baseline or separation between the telescopes. The staircase mirror was manufactured at Philips Research Laboratories (Eindhoven, The Netherlands) with a diamond turning machine using a $3-\mu \mathrm{m}$ chisel. For the chosen step width of $1339 \pm 6 \mu \mathrm{m}$ both the on- and the off-axis beams are focused close to the center of their respective steps. The corresponding depth of the steps is $3 \pm 0.2 \mu \mathrm{m}$.

The staircase mirror holder can be rotated such that the steps can be set either perpendicular to the baseline to correct the external optical path, or parallel to it, which corresponds to an uncorrected situation (i.e., no staircase mirror).

The light is collimated again before it enters the delay line-beam combiner block. Two mirrors on top of a piezo stage, set in one of the interferometer arms, provide a delay line to modulate the optical path. The beams overlap at a beam splitter-combiner cube. The two outputs of the beam splitter are detected by a low-noise photometric detector and a CCD camera that measures the modulated fringes and controls the pupil position, respectively.

\section{Experimental Results}

\section{A. Assembly, Alignment, and Calibration}

The setup was assembled starting from the star simulator; then we added both interferometers arms, the delay line mirrors, and the beam combiner. A He-Ne laser set before the star mask was used for the alignment. The piezo stage of the delay line was actuated with a triangle function of $4-\mathrm{V}$ amplitude, equivalent to a modulation of the path of approximately $9 \mu \mathrm{m}$. The fringes produced by the coherent interference of the beams were detected with a photodetector, as was the intensity of each beam for calibration of the visibility. The maximum visibility obtained with the laser was $0.92 \pm 0.02$, as shown in the calibrated fringe pattern in Fig. 11. Higher-contrast fringes should be expected from a monochromatic point source. The
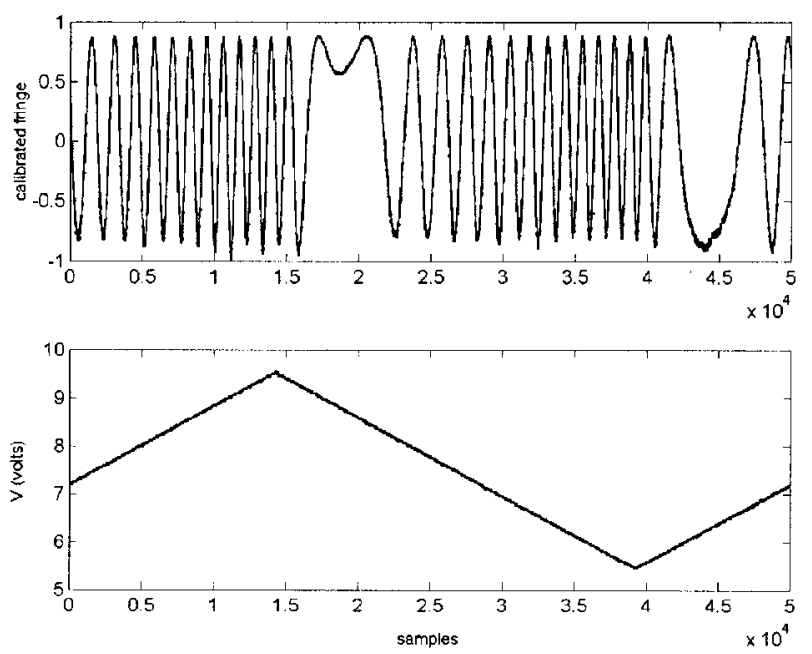

Fig. 11. Experimental laser fringes used for alignment and calibration.

limited observed contrast could have either of two causes: misalignment or the fact that the observed source is partially resolved by the baseline. To calibrate the instrument for the white-light measurements it is necessary to know whether the object observed is a point source. Direct measurements of the distance from the star mask to the collimating lens indicate a distance of $480 \mathrm{~mm}$, i.e., $20 \mathrm{~mm}$ shorter than the focal length of the lens. Consequently the collimating lens is not relaying the star mask at infinity but producing a virtual image of a star with a $0.9 \times 10^{-5} \mathrm{rad}$ angular diameter. The visibility at $632 \mathrm{~nm}$, observed with a baseline of 30 $\mathrm{mm}$, is expected to be 0.95 . Given this number, the residual reduction would be attributed to alignment or surface errors. The total reduction that is due to these factors is $\sim 3 \%$. For a partially resolved source the visibility depends on the wavelength; given the measured values of the visibility with the laser radiation, the expected visibility for the white-light source, with a central wavelength of $575 \mathrm{~nm}$, is $\sim 0.90$.

\section{B. Dispersion Correction}

When we measured the white-light fringes, first results showed that fringes for the on-axis and off-axis stars were observed simultaneously when the dividing lines of the steps of the staircase mirror were perpendicular to the baseline (i.e., with correction of the OPD). But the contrast was 0.26 , which is quite a low value. The reason for such low contrast compared with the contrast measured with the laser is attributed to dispersion produced by the various optical elements. There are three achromats in each arm of the interferometer, and every achromat is composed of two different types of glass, with a tolerance error of $\sim 100 \mu \mathrm{m}$. The beam splitter used to combine the light is a cube made from BK7 glass, with a tolerance error, according to the manufacturer, of $\sim 200 \mu \mathrm{m}$. When the optical path through the various glasses is 

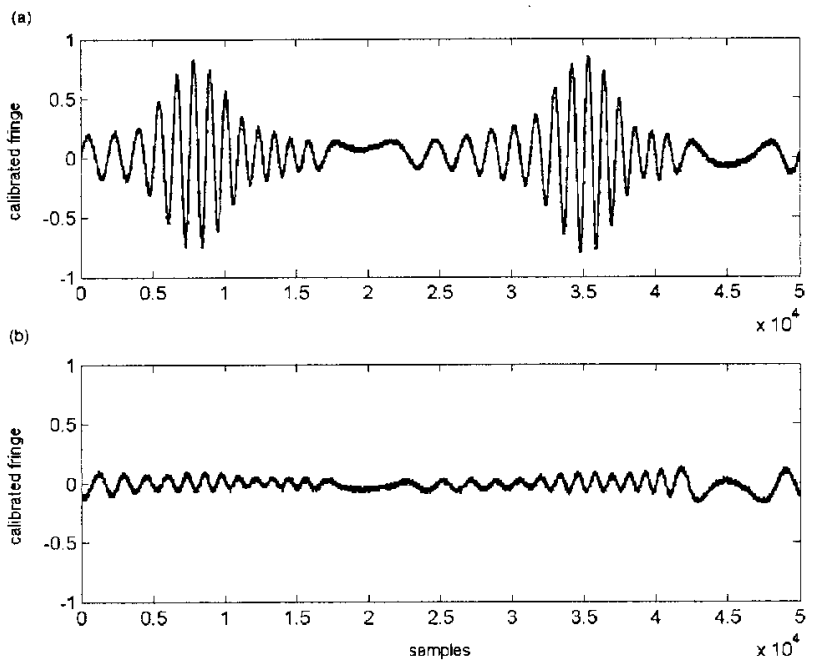

Fig. 12. White-light fringes when the mirror has steps parallel to the baseline: (a) on-axis calibrated fringe, (b) off-axis calibrated fringe. The off-axis star fringe is out of the interferometric field of view; its peak is not observable.

larger in one arm than in the other, the zero-path position for every wavelength is different, and the result is a broader set of fringes with lower contrast. The different types of glass present in the setup are BK7, F2, SF2, and SF5. To correct the dispersion we inserted two types of glass that we expected to make larger contributions to the dispersion (BK7 and F2), into the interferometer's arms. By tilting the glass plates and observing the fringes, we obtained a visibility after optimization of $0.82 \pm 0.02$.

\section{Measurements}

After correction for the dispersion, we measured the fringes corresponding to the four stars in the star mask. In the first set of data the staircase mirror was placed with the steps parallel to the baseline, such that no correction of the external OPD was introduced. The calibrated fringes, obtained with a total scanning distance of $9 \mu \mathrm{m}$, are shown in Fig. 12. The fringe from the on-axis star [Fig. 12(a)] has a visibility of $0.82 \pm 0.02$. The peak of the fringe from the off-axis star is not observed, as it is not in the field of view of the interferometer, and the visibility was $0.14 \pm 0.02$ [Fig. 12(b)]. Similar results were found with the other two on- and off-axis stars. In the second set, we placed the mirror with the steps perpendicular to the baseline, so correction of the external OPD did take place. The results are shown in Fig. 13. The visibility calculated from the calibrated fringe of the two on-axis stars is again $0.82 \pm 0.02$ [Figs. 13(a) and $13(\mathrm{c})]$. The visibility of the two off-axis stars is $0.74 \pm 0.02$ [Figs. 13(b) and 13(d)]. This value, compared with the visibility of the off-axis star without correction [Fig. 12(b)], clearly demonstrates the effect of the OPD correction that is due to the steps.

One can calculate the relative angle, $\varphi$, that gives the position of the off-axis star with respect to the
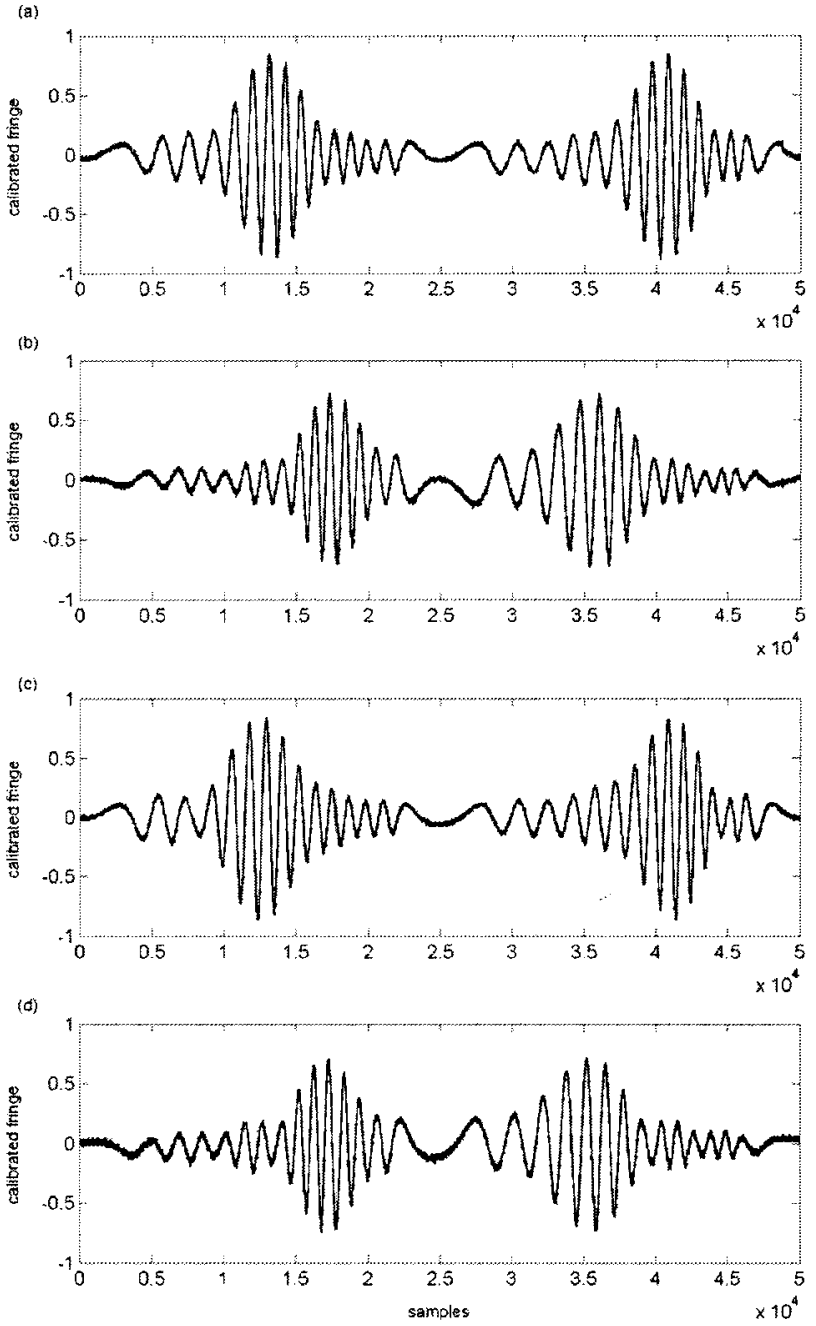

Fig. 13. White-light fringes when the steps of the mirror are perpendicular to the baseline: (a), (c) calibrated fringes from the on-axis stars; (b), (d) calibrated fringes from the off-axis stars. The interferometric field of view has been extended, and the peaks of the fringes of the off-axis stars are observed.

on-axis star by measuring the distance from the peak of the off-axis fringe pattern to the peak of the on-axis pattern, $n_{p-p}$, and knowing in which step the off-axis star is focused:

$$
\varphi=\frac{1}{B}\left(2 n d \cos \alpha+n_{p-p}\right)
$$

where $B$ is the baseline, $d$ is the depth of the steps, $\alpha$ is the angle that the mirror forms with the optical axis, and $n_{p-p}$ can be either positive or negative. In our case the relative angular separation between the stars measured from the fringe pattern is $(3.3 \pm 0.1) \times 10^{-4} \mathrm{rad}$, which agrees closely with the calculation of the angular position by direct measurement of the experimental parameters.

The visibility of the off-axis fringes is not equal to the visibility of the on-axis fringe pattern, again because of the dispersion effects. For the data shown in 

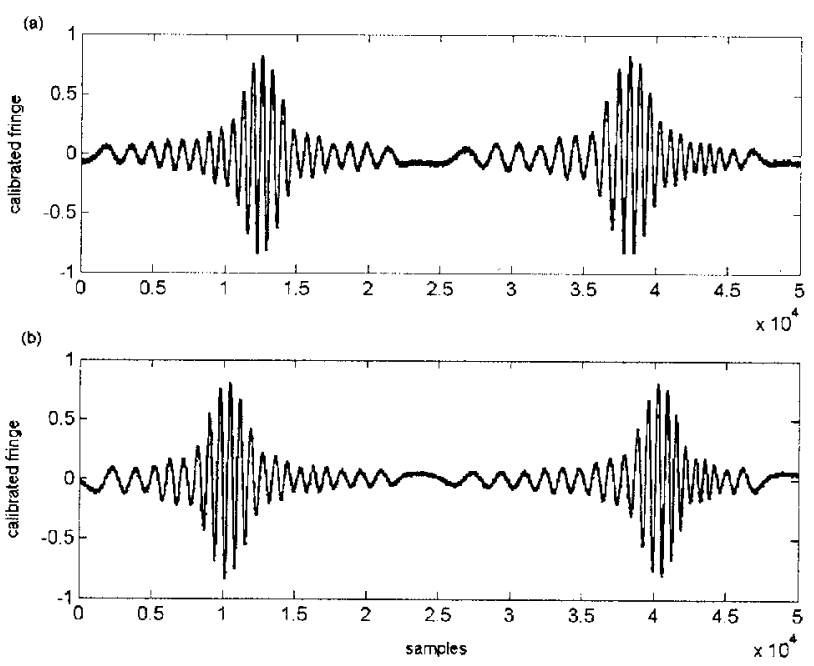

Fig. 14. (a), (b) White-light fringes for two stars, both symmetric with respect to the optical axis; hence the same dispersion correction is applied, and they show similar contrast.

Fig. 13 we set the on-axis stars at the centers of the achromats, and the dispersion correction was optimized for this direction. Therefore the off-axis stars hit the achromats off axis, and the dispersion correction is not optimum. By moving the stars in such a way that they hit the achromats symmetrically, we observed the same visibility, $0.80 \pm 0.02$, in both cases, as shown in Fig. 14, where the stars' calibrated fringes are plotted.

\section{Conclusions}

We have presented a new approach to achieving a wide field of view in optical interferometry. The main advantages of our approach are that a wide field of view is achieved in one shot and that the flexible system is compatible with image or pupil-plane combination interferometers. The compatibility with pupil-plane interferometers permits beam combination with a signal-to-noise ratio larger than the homothetic mapping solution with Fizeau or imaging Michelson-type interferometers. Experimental results have been shown that prove the feasibility of the concept. Fringes from an off-axis star separated 1.07 arc min from an on-axis reference star have been obtained simultaneously and with a contrast similar to that of the on-axis star. The relative angular separation of the off-axis star has been retrieved and corresponds to the expected value. In this paper we analyzed the correction of the OPD for the simple case of a few stars focused on different steps of a staircase mirror. It is also important to investigate what happens to the fringe pattern when a star is focused on the edge of a step. It turns out that, even though the OPD correction is discontinuous, the visibility can be completely recovered, so the information is not lost. More details about this issue have been studied, and we intend to report them. The experimental results presented in this paper were ob- tained with a mirror of fixed shape, but, as has been explained, an actuated mirror is required if the system is used for astronomical observations. The mirror could consist of an array of parallel strips, each of them mounted upon a piezo stage, independently controlled, to actively correct the OPD. Future research will focus on the design and test of such an actuated staircase mirror.

The authors thank the Knowledge Center for Aperture Synthesis, a collaboration of the Netherlands Organisation for Applied Scientific Research TNO and the Delft University of Technology, for financial support. We also thank Philips Research Laboratories for manufacturing the staircase mirror. We are grateful to Aad van der Lingen for his very helpful technical support during the assembly and alignment of the setup.

\section{References}

1. A. Labeyrie, "Stellar interferometry methods," Ann. Rev. Astron. Astrophys. 16, 77-102 (1978).

2. M. Tallon and I. Tallon-Bosc, "Beam combination for wide field imaging [invited]," in Very High Angular Resolution Imaging, Int. Astron. Union Symp. 158, 83-90 (1994).

3. J. M. Beckers, "Field of view considerations for telescope arrays," in Advanced Technology Optical Telescopes III, L. D. Barr, ed., Proc. SPIE 628, 255-260 (1986).

4. J. M. Beckers, "The VLT interferometer. III. Factors affecting wide field-of-view operation," in Advanced Technology Optical Telescopes IV, Proc. SPIE 1236, L.D. 379-389 (1990).

5. G. Rousset, L. M. Mugnier, F. Cassaing, and B. Sorrente, "Imaging with multi-aperture optical telescopes and an application," C. R. Acad. Sci. Paris IV 2, 17-25 (2001).

6. C. A. Haniff, "Practical considerations for imaging interferometry," in Working on the Fringe: Optical and IR Interferometry from Ground and Space, Astron. Soc. Pac. Conf. Ser. 194, 230-240 (1999).

7. O. Lardiere, A. Labeyrie, D. Mourard, P. Riaud, L. Arnold, J. Dejonghe, and S. Gillet, "VIDA (VLTI Imaging with a densified array), a densified pupil combiner proposed for snapshot imaging with the VLTI," in Interferometry for Optical Astronomy II, W. Traub, ed., Proc. SPIE 4838, 1018-1027 (2003).

8. A. Labeyrie, "Resolved imaging of extra-solar planets with future 10-100-km optical interferometric arrays," Astron. Astrophys. Suppl. Ser. 118, 517-524 (1996).

9. E. Pedretti, A. Labeyrie, L. Arnold, N. Thureau, O. Lardiere, A. Boccaletti, and P. Riaud, "First images on the sky from a hyper telescope,” Astron. Astrophys. Suppl. Ser. 147, 285-290 (2000).

10. M. Schoeller, R. Wilhelm, and B. Koehler, "Modeling the imaging process in optical stellar interferometers," Astron. Astrophys. Suppl. Ser. 144, 541-552 (2000).

11. A. Glindemann, R. Abuter, F. Carbognani, F. Delplancke, F. Derie, A. Gennai, P. Gitton, P. Kervella, B. Koehler, S. Leveque, S. Menardi, A. Michel, F. Paresce, T. P. Duc, A. Richichi, M. Scholler, M. Tarenghi, A. Wallander, and R. Wilhelm, "The VLT interferometer," C. R. Acad. Sci. Paris IV 2, 57-65 (2001).

12. A. R. Thompson, J. M. Moran, and G. W. Swenson, Interferometry and Synthesis in Radio Astronomy (Krieger, Malabar, Fla., 1991).

13. I. Montilla, E. J. Bakker, S. F. Pereira, and J. J. M. Braat, "A new concept for wide field imaging," in Interferometry for $\mathrm{Op}$ tical Astronomy II, W. Traub, ed., Proc. SPIE 4838, 416-424 (2003). 